\title{
Sporadic Creutzfeldt-Jakob disease: A clinical approach of a small case series and literature review
}

\author{
Sebastian Andone' ${ }^{1}$, Sanda Petrutiu' ${ }^{1}$ Zoltan Bajko ${ }^{1,2}$, Anca Motataianu' ${ }^{1,2}$, \\ Smaranda Maier ${ }^{1,2}$, Ioan Macavei ${ }^{1}$, Adina Stoian ${ }^{1,3}$, Adrian Balasa ${ }^{4,5}$, Rodica Balasa ${ }^{1,2}$ \\ ${ }_{1}^{1}$ st Neurology Clinic, Mures County Clinical Emergency Hospital, Romania \\ ${ }^{2}$ Department of Neurology, University of Medicine and Pharmacy, Targu Mures, Romania \\ ${ }^{3}$ Department of Physiopathology, University of Medicine and Pharmacy, Targu Mures, Romania \\ ${ }^{4}$ Department of Neurosurgery, University of Medicine and Pharmacy, Targu Mures, Romania \\ ${ }^{5}$ Neurosurgery Clinic, Mures County Clinical Emergency Hospital, Romania
}

\begin{abstract}
Introduction. Creutzfeldt-Jakob Disease (CJD) is a very rare neurodegenerative disease with no cure that always leads to death. It is the most frequent prion disease and has multiple recognized forms, from which the sporadic form ( $\mathrm{SCJD}$ ) is the most common. The defining histological characteristic of CJD is the accumulation of the abnormal prion protein (PrPSc) along with neuronal loss and spongiform vacuolation. Typical neurological signs found in SCJD are rapidly progressive dementia, myoclonus, visual disturbances, pyramidal signs, extrapyramidal signs, behavioral changes and akinetic mutism.

Material and method. We performed a retrospective study over the 2007-2017 period that included five cases of patients diagnosed with probable sCJD from our department.

Results. The most common findings in the patients' MRI were cerebral atrophy and ventricular enlargement, but we also found typical MRI changes such as increased T2 and FLAIR signal intensity in the head of the caudate nucleus, putamen and cerebral cortex. Repeated electroencephalograms showed typical SCJD patterns like periodic sharp wave complexes, mostly with biphasic or triphasic aspect. A complex differential diagnosis and numerous routine investigations (CBC, biochemical analysis, autoimmune panel, HIV antibodies, VDRL, anti-Borrelia antibodies, anti-TPO antibodies, anti-neuronal antibodies, B12 vitamin) were performed, without indicating an alternative diagnosis. All the patients died several months after the onset of symptoms.

Conclusions. Given the rarity of the disease and the broad differential diagnosis, CJD proves to be a diagnostic challenge. In the absence of an alternative diagnosis, the association of rapidly progressive dementia, myoclonus, visual and cerebellar signs are highly suggestive for SCJD.
\end{abstract}

Keywords: sporadic Creutzfeldt-Jakob disease, prion disease, rapidly progressive dementia

\section{INTRODUCTION}

Creutzfeldt Jakob Disease (CJD) is a very rare degenerative disease, with a rapidly progressive evolution towards dementia and death, being the most frequent of the human prion diseases.

There are several recognized forms of CJD like sporadic (sCJD), familial (fCJD), iatrogenic (iCJD) and variant form of CJD (vCJD), the sporadic form being the most common amongst them $(85-95 \%$ of cases) $(1,2)$.
CJD's defining histological characteristics are spongiform vacuolation, neuronal loss without inflammation and accumulation of the abnormal prion protein, together known as "status spongiosus" (3).

Several molecular subtypes of SCJD have been described and are associated with PRNP gene codon 129 and PrPSc (pathologic prion protein). The most common phenotype is MM1 which correlates with the classic SCJD with periodic sharp wave 
complexes on the electroencephalogram, accounting for about $70 \%$ of cases (4-7).

The most typical neurological signs found in SCJD are rapidly progressive dementia, myoclonus, behavioral abnormalities, memory difficulties, visual disturbances and akinetic mutism, death usually occurring within one year of symptom onset $(8,9)$.

We present five cases of patients from our department that have been diagnosed with probable sCJD.

In our department, over a period of 10 years, from a total of 26124 cases, only $0.02 \%$ represent patients diagnosed with SCJD, being an extremely rare pathology.

\section{MATERIAL AND METHOD}

This was a retrospective study in the 2007- 2017 period, that included five patients admitted in the Department of Neurology of Targu Mures Emergency County Hospital, diagnosed with probable sCJD, according to CDC's criteria for CJD diagnosis (Table 1).
All our cases had no family history related to CJD and did not receive blood transfusion or had any invasive maneuvers performed, all being sporadic cases of the disease.

Our five patients presented a broad spectrum of clinical features which are summarized in Table 2.

We performed multiple serological tests such as $\mathrm{CBC}$, biochemical analysis, autoimmune panel, HIV antibodies, VDRL, anti-Borrelia antibodies, anti-TPO antibodies, anti-neuronal antibodies, B12 vitamin, all within normal range. The routine investigations did not indicate an alternative diagnosis.

We ruled out other dementias, like Alzheimer disease, dementia with Lewy bodies and frontotemporal dementias, as well as any other causes of subacute dementias such as electrolytes imbalance, B12 vitamin deficit, hepatic encephalopathy, uremic encephalopathy, neurosyphilis, HIV infection, Hashimoto's thyroiditis and related encephalopathy.

TABLE 1. CDC's Diagnostic Criteria for Creutzfeldt-Jakob Disease (CJD), 2010 (10)

\begin{tabular}{|c|c|}
\hline \multicolumn{2}{|l|}{ 1. Sporadic CJD } \\
\hline Definite SCJD & $\begin{array}{l}\text { - Diagnosed by standard neuropathological techniques; and/or immunocytochemically; and/or Western blot } \\
\text { confirmed protease-resistant PrP; and /or presence of scrapie-associated fibrils. }\end{array}$ \\
\hline Probable sCJD & $\begin{array}{l}\text { - Rapidly progressive demen a } \\
\text { - At least two out of the following four clinical features: } \\
\text { - Myoclonus } \\
\text { - Visual or cerebellar signs } \\
\text { - Pyramidal/extrapyramidal signs } \\
\text { - Akine c mu sm } \\
\text { - A posi ve result on at least one of the following laboratory tests: } \\
\text { - a typical EEG (periodic sharp wave complexes) during an illness of any dura on; and/or } \\
\text { - a posi ve 14-3-3 cerebrospinal fluid (CSF) assay in pa ents with a disease dura on of less than } 2 \text { years } \\
\text { - Magne c resonance imaging (MRI) high signal abnormali es in caudate nucleus and/or putamen on diffusion- } \\
\text { weighted imaging (DWI) or fluid a enuated inversion recovery (FLAIR) } \\
\text { - Without rou ne inves ga ons indica ng an alterna ve diagnosis }\end{array}$ \\
\hline Possible sCJD & $\begin{array}{l}\text { - Progressive demen a } \\
\text { - At least two out of the following four clinical features: } \\
\text { - Myoclonus } \\
\text { - Visual or cerebellar signs } \\
\text { - Pyramidal/extrapyramidal signs } \\
\text { - Akine c mu sm } \\
\text { - The absence of a posi ve result for any of the three laboratory tests that would classify a case as "probable" } \\
\text { - Dura on of illness less than two years } \\
\text { - Without rou ne inves ga ons indica ng an alterna ve diagnosis }\end{array}$ \\
\hline \multicolumn{2}{|c|}{ 2. latrogenic CJD } \\
\hline \multicolumn{2}{|c|}{$\begin{array}{l}\text { - Progressive cerebellar syndrome in a recipient of human cadaveric-derived pituitary hormone; or sporadic CJD with a recognized } \\
\text { exposure risk, e.g., antecedent neurosurgery with dura mater implanta on. }\end{array}$} \\
\hline \multicolumn{2}{|l|}{ 3. Familial CJD } \\
\hline \multicolumn{2}{|c|}{$\begin{array}{l}\text { - Definite or probable CJD plus definite or probable CJD in a first degree rela ve; and/or Neuropsychiatric disorder plus disease-specific } \\
\text { PrP gene muta on. }\end{array}$} \\
\hline
\end{tabular}


TABLE 2. Summary of symptoms

\begin{tabular}{|c|c|c|c|}
\hline & Prodromal symptoms & Early signs & Late signs \\
\hline Case 1 & $\begin{array}{l}\text { - Behavioral changes } \\
\text { - Depression }\end{array}$ & $\begin{array}{l}\text { - Cogni ve deteriora on } \\
\text { - Rigidity }\end{array}$ & $\begin{array}{l}\text { - Generalized myoclonus } \\
\text { - Severe rigidity } \\
\text { - Akinesia } \\
\end{array}$ \\
\hline Case 2 & $\begin{array}{l}\text { - Behavioral changes } \\
\text { - Fa gue } \\
\text { - Dizziness }\end{array}$ & $\begin{array}{l}\text { - Visual disturbances } \\
\text { - Rapidly progressive demen a } \\
\text { - Ataxia }\end{array}$ & $\begin{array}{l}\text { - Generalized myoclonus } \\
\text { - Severe demen a } \\
\text { - Rigidity } \\
\text { - Pyramidal signs } \\
\text { - Extrapyramidal signs }\end{array}$ \\
\hline Case 3 & $\begin{array}{l}\text { - Behavioral changes } \\
\text { - Dizziness }\end{array}$ & $\begin{array}{l}\text { - Demen a } \\
\text { - Ataxia } \\
\text { - Visual disturbances } \\
\text { - Severe confusion }\end{array}$ & $\begin{array}{l}\text { - Generalized myoclonus } \\
\text { - Severe ataxia } \\
\text { - Severe demen a } \\
\text { - Heteroagresivity } \\
\text { - Disarthria }\end{array}$ \\
\hline Case 4 & - Behavioral changes & $\begin{array}{l}\text { - Progressive demen a Bradylalia } \\
\text { - Bradykinesia }\end{array}$ & $\begin{array}{l}\text { - Generalized myoclonus } \\
\text { - Extrapyramidal signs } \\
\text { - Rigidity } \\
\text { - Severe demen a }\end{array}$ \\
\hline Case 5 & $\begin{array}{l}\text { - Behavioral changes } \\
\text { - Poor memory }\end{array}$ & $\begin{array}{l}\text { - Demen a } \\
\text { - Agnosia } \\
\text { - Apraxia } \\
\text { - Ataxia } \\
\text { - Visual disturbances } \\
\text { - Agita on }\end{array}$ & $\begin{array}{l}\text { - Generalized myoclonus } \\
\text { - Pyramidal signs } \\
\text { - Extrapyramidal signs } \\
\text { - Rigidity } \\
\text { - Akinesia } \\
\text { - Severe demen a }\end{array}$ \\
\hline
\end{tabular}

\section{Case 1}

A 36 year old woman, presented one month prior to admission, depression, anxiety, for which she was evaluated multiple times by psychiatric and neurological services. She was admitted in our clinic through the Emergency Unit where she was brought for an akinetic syndrome. Her past medical history was not significant.

The neurological exam revealed generalized myoclonus, rigidity, without the presence of pyramidal signs. A series of cerebral MRI examinations were performed, all of them showing moderate diffuse cerebral atrophy in both hemispheres with the enlargement of the ventricles. An infectious disease consultation put the diagnosis of subacute encephalopathy of unknown etiology. Her electroencephalogram showed theta and delta activity with paroxistic sharp wave complexes which is compatible with the encephalopathy diagnosis. The patient died 3 months after her hospitalization.

\section{Case 2}

A 69 year old woman, presented visual disturbances and rapidly progressive cognitive deterioration in the last two months, and generalized myoclonus for which she was admitted in our service.

The neurological exam revealed spastic tetraparesis, muscular rigidity, myoclonus, bilateral Babinski sign and severe cognitive deterioration.
The electroencephalogram showed theta and delta polymorph background waves with triphasic sharp wave complexes that appear periodically at variable time intervals. The cerebral MRI showed increased bilateral T2 and FLAIR signal intensity in the head of the caudate nucleus, putamen and cerebral cortex, which is a typical image for SCJD.

The patient's condition aggravated, with an evolution toward death within 3 months after being discharged.

\section{Case 3}

A 64 year old woman, presented balance disorder, movement difficulties, rapidly cognitive deterioration for over a month, associated with visual disturbances, confusion, temporal and spatial disorientation in the last week before admission.

The neurological exam revealed severe ataxia, generalized myoclonus, visual agnosia, dysarthria without any pyramidal signs.

The cerebral MRI showed increased FLAIR signal intensity in the cerebral cortex, mostly occipital and temporal, and the enlargement of ventricles.

A series of electroencephalograms were performed, showing irregular theta waves and periodic sharp wave complexes with biphasic and mostly triphasic aspect.

The patient died 4 months after her hospitalization. 


\section{Case 4}

A 64 year old man, was referred to our clinic presenting tremor, generalized myoclonus, temporal and spatial disorientation, cognitive deterioration that aggravated progressively in the last 5 months.

The neurological exam showed hypomimia, decreased blink rate, parkinsonian gait, myoclonus, muscular hypertonia, without any pyramidal signs, bradypsychia, bradylalia, bradykinesia, and a severe cognitive deterioration.

The patient's electroencephalography showed on the fronto-parietal derivations polymorph theta and delta waves and irregular triphasic sharp wave complexes.

The cerebral MRI showed bilateral cortical atrophy without other pathological findings.

The patient died 3 months after being discharged.

\section{Case 5}

A 68 years old male patient, presented with generalized myoclonus, visual disturbances, memory loss, cognitive deterioration with a subacute evolution of over 2 months.

The neurological exam showed agnosia, apraxia, difficulty of movement due to severe extrapyramidal hypertonia, generalized myoclonus, rapidly progressive cognitive deterioration, pyramidal signs, bradypsychia, bradylalia.

The cerebral MRI showed in both hemispheres cortical atrophy and multiple spots in the periven-

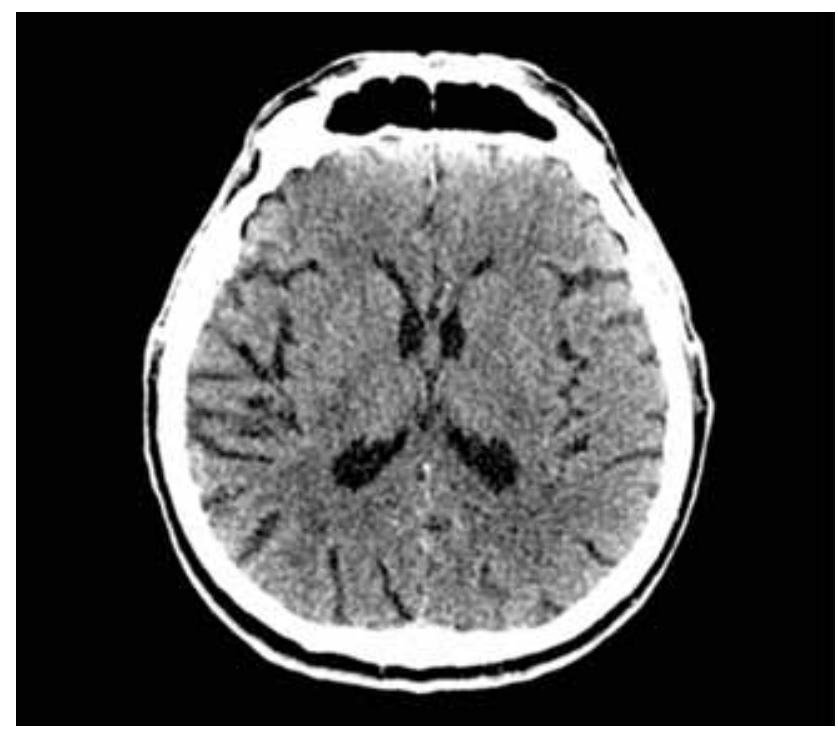

FIGURE 1. CT-scan (day 1 of hospitalization) of case 5

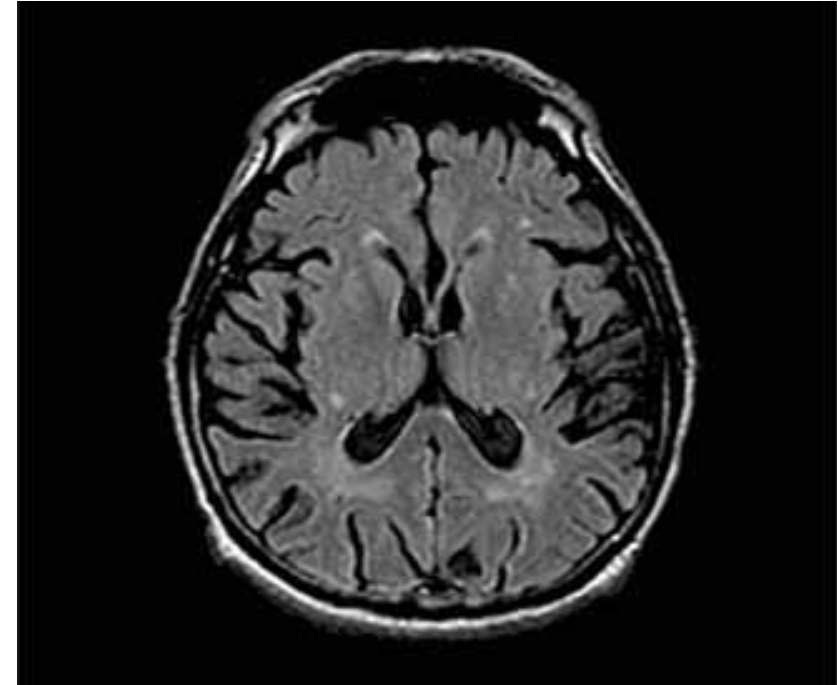

FIGURE 2. IRM - FLAIR (day 21 of hospitalization) of case 5

tricular areas with an increased T2 and FLAIR signal intensity.

A series of electroencephalograms were performed showing triphasic periodic sharp wave complexes.

The patient died 4 weeks after hospitalization.

\section{DISCUSSIONS}

There are five major classes of prion disease that had been reported and recognized in humans. These include CJD, Gerstmann-Straussler-Scheinker syndrome (GSS), fatal insomnia (FI), new variant CJD (nvCJD) and Kuru disease (3).

As mentioned in the introduction, CJD consists of different forms, with different etiologies. While vCJD has been linked to the consumption of bovine meat, fCJD is determined by an autosomal dominant mutation within the gene encoding PrP, sCJD is caused either by spontaneous production of $\operatorname{PrP}$, either by a somatic mutation of $\operatorname{PrP}$, or by unidentified environmental prion exposure (11).

sCJD is a very rare disease, with an incidence of approximately 1 case per 1,000,000 population per year. $(1,2)$

In the last 10 years the incidence of SCJD in Mures County was approximately 0.9 cases per $1,000,000$ population per year. Compared to other countries such as Canada, where the incidence rate in the last 10 years was 1.44 (1.11-1.81) our incidence is much smaller (12).

The mean age of onset for SCJD is between 55 and 62 years, but there are also rare cases of patients younger than 40 years old or older than 80 


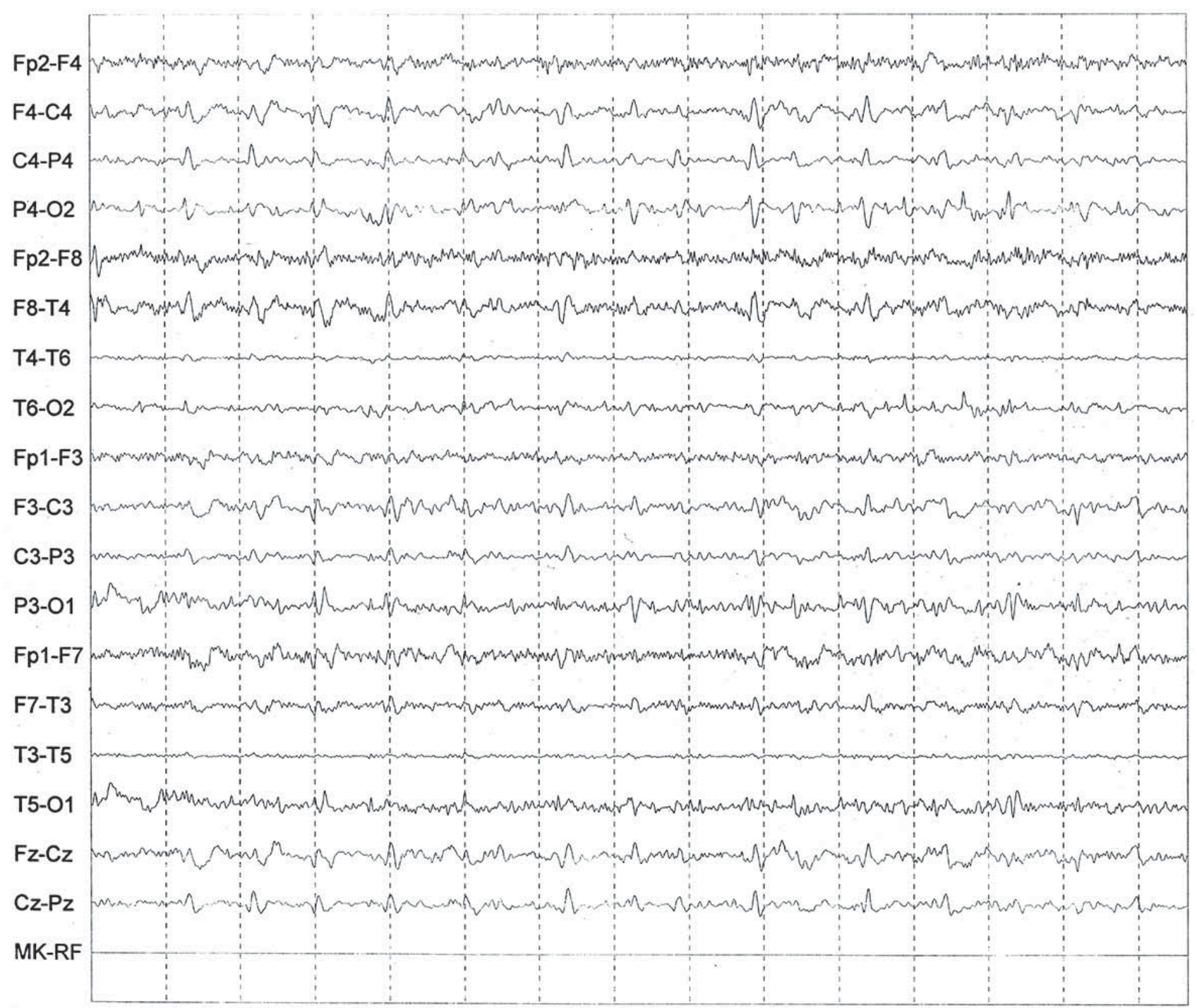

FIGURE 3. Electroencephalogram with periodic charges of a patient with probable sCJD

years old (13). In our patients the mean age was 60 years old with the minimum age being 36 years old while the maximum was 69 years old. The defining characteristic of CJD is its rate of progression, differentiating it from other neurodegenerative diseases and other dementias. It should be noted that sCJD has a more rapid fatal course (mean duration of 8 months) than fCJD which can prolong over a few years (mean duration of 26 months) (3).

The prodromal symptoms most commonly described in literature are: headache, dizziness, paresthesias, fatigue, or behavioral changes (3). All our patients presented behavioral changes, being one of the main reason their families sought medical assistance initially.

Because such symptoms can be overlooked by the patients and their families, the first signs of the disease could be any of the symptoms included in the typical clinical triad of CJD, such as cognitive disturbances, ataxia and myoclonus (3). Initially the myoclonus may begin focally in a single limb, however in the late stages of the disease it becomes generalized and along with akinetic mute state they represent the end stage of the disease.

As early sign of the disease, a variable degree of cognitive deterioration was found in all of the cases. Three out of five patients presented visual disturbances, as well as ataxia.

A similar feature between our cases was the generalized myoclonus as a late sign of the disease, as well as the aggravation of the early symptoms. Severe dementia and rigidity were also present as late features of the disease. Two of our cases presented akinesia in the late stages of the disease.

MRI can be very helpful in the diagnosis of CJD. While all the patients presented generalized 
atrophy and ventricles enlargement, which can be a characteristic found in early to late SCJD, only two of them had characteristic MRI changes which fulfilled CJD diagnostic criteria. Repeated neuroimaging investigations showed a rapidly progressive cortical atrophy (Fig. 1, Fig. 2).

The electroencephalogram can provide evidence for CJD such as periodic sharp wave complexes (PSWC) that can be observed in $67-95 \%$ of patients with SCJD (14), the most characteristic pattern being synchronous bi- or triphasic sharp wave complexes. It should be noted that a single EEG may not capture the discharges pattern thus it should be repeated over the course of the disease, because it typically has a progressive evolution. We observed this mechanism in our patients, to which we performed multiple EEG examinations, ultimately being able to capture sCJD typical patterns (Fig. 3).

The 14-3-3 protein can be found in SCJD as well as in other neurodegenerative diseases, as it is released in the process of neural cell death. Literature findings show that early in the course of disease the test may provide falsely negative results. However, in our patients we could not perform this examination from different reasons.

An extensive differential diagnosis was performed with other neurodegenerative diseases, dementias, and reversible causes of subacute dementias as mentioned before. Hashimoto's thyroiditis and related encephalopathy has been reported as a masquerader of prion disease, and it should be mentioned that a normal TSH level does not exclude this disease, but in case of a suspicion antithyroglobulin and antithyroperoxidase antibodies should be performed. This disease is responsive to treatment and the condition can be completely re- versed when under correct treatment, so it is extremely important to consider it in the differential diagnosis (15).

During hospitalization all of the patients received symptomatic treatment such as sedatives, antiepileptic drugs, anti-inflammatory drugs, vitamins and gastric protection drugs as well as chronic treatment for associated diseases.

The mean survival time from the onset of symptoms has been reported in literature to be around 8 months (3). In our case, the mean survival time was 5 months, minimum survival time being 3 months while the maximum survival time being 8 months.

Due to MRI and EEG findings, the associated symptoms and a thorough differential diagnosis, we placed our five patients into the probable sCJD group.

\section{CONCLUSIONS}

sCJD proves to be a diagnostic challenge for any physician due to its progressive symptoms and a broad differential diagnosis. Even if the disease is incurable and has an inevitable evolution towards death it is of utmost importance to make a correct diagnosis as early as possible, because some of the differential diagnosis diseases are treatable.

However, the association of rapidly progressive dementia, myoclonus, visual and cerebellar signs are highly suggestive for SCJD in the absence of another possible diagnosis.

\section{Acknowledgement}

This publication was supported by the internal research grant of the University of Medicine and Pharmacy Targu Mures, grant nr. 18/2015.

Conflict of interest: none declared

\section{REFERENCES}

1. Masters C.L., Harris J.O., Gajdusek D.C. et. al. Creutzfeldt-Jakob Disease: patterns of worldwide occurrence and the significance of familial and sporadic clustering. Ann Neurol 1979; 5;177

2. Ladogana A., Puopolo M., Croes E.A. et. al. Mortality from Creutzfeldt-Jakob disease and related disordes in Europe, Australia and Canada. Neurology 2005; 64; 1586.

3. Larry E. Davis et al. Continuum. Lifelorng learning in Neurology. Infectious Disease. Volume 8 Number 3 June 2002: 130-145.

4. Parchi Piero et al. „Molecular basis of phenotypic variability in sporadc creudeldt-jakob disease." Annals of neurology 39.6 (1996): 767-778.

5. Parchi Piero et al. „Classification of sporadic Creutzfeldt-Jakob disease based on molecular and phenotypic analysis of 300 subjects." Annals of neurology 46.2 (1999): 224-233.

6. Lewis V. et al. „Australian sporadic CJD analysis supports endogenous determinants of molecular-clinical profiles." Neurology 65.1 (2005): 113-118.

7. Collins S.J. et al. „Determinants of diagnostic investigation sensitivities across the clinical spectrum of sporadic CreutzfeldtJakob disease." Brain 129.9 (2006): 2278-2287.

8. Haywood A.M. Transmissible spongiform encephalopathies. N. Engl J Med 1997; 337:1821 
9. Rabinovici G.D., Wang P.N., Levin J. et al. First symptoms in sporadic Creutzfeldt-Jakob disease. Neurology 2006; 66; 286

10. CDC's Diagnostic Criteria for Creutzfeldt-Jakob Disease (CJD), 2010, http://cdc.gov/ncidod/dvrd/cjd/diagnostic_criteria.html

11. Hill, Andrew F. et al. "Molecular classification of sporadic Creutzfeldt-Jakob disease." Brain 126.6 (2003): 1333-1346.

12. Public Health Agengy of Canada www.phac-aspc.gc.ca/hcai-iamss/ cjd-mcj/cjdss-ssmcj/stats-eng.php

13. Brown P. et al. „The epidemiology of Creutzfeldt-Jakob disease Conclusion of a 15-year investigation in France and review of the world literature." Neurology 37.6 (1987): 895-895.
14. Steinhoff B.J., Racher S., Herrendorf G. el al. Accuracy and reliability of periodic sharp wave complexes in Creutzfeldt-Jakob disease. Arch Neurol 1996 53-162

15. Seipelt M. et al. "Hashimoto's encephalitis as a differential diagnosis of Creutzfeldt-Jakob disease." Journal of Neurology, Neurosurgery \& Psychiatry 66.2 (1999): 172-176. 